\title{
稠密平分子图与表压缩问题的 近似算法*
}

\author{
徐大川 ${ }^{* *}$ \\ (北京工业大学应用数理学院, 北京 100022) \\ 韩继业 \\ (中国科学院数学与系统科学研究院应用数学研究所, 北京 100080) \\ 杜东雷
}

(Faculty of Administration, University of New Brunswick, P. O. Box 4400, Fredericton, NB E3B 5A3, Canada)

摘要给出两个 NP 问题 (稠密平分子图和表压缩) 的改进的近似算法. 基于 半定规划 (SDP) 松驰和巧妙的舍入技巧, 首先给出稠密平分子图问题 (DSP) 的 0.5982-近似算法, 表压缩问题 (TCP) 的 0.5970-近似算法. 然后, 通过增加三角 不等式得到更紧的 SDP 松驰, 把前面的比值分别改进到 0.6243 和 0.6708. 针对 $\mathrm{TCP}$ 得到的结果改进了简单贪婪算法的 0.5 近似比, 因此回答了 Anderson 提出的 未解决问题.

\section{关键词稠密平分子图问题 表压缩问题 半定规划 近似比}

\section{1 引言}

我们考虑两个相关的 NP 问题: 稠密平分子图问题和表压缩问题, 分别定义 如下:

定义 1.1(稠密平分子图问题 (DSP)) 设 $G=(V, E)$ 是一个无向图, 顶点个数 $n=|V|$ 为偶数. 对边定义一个对称非负的权函数 $w: E \rightarrow \mathbb{R}^{+}$. 我们要寻找一个 顶点个数为 $n / 2$ 的子集 $S \subset V$, 使得由 $S$ 导出的子图的所有权和最大.

2005-03-31 收稿

* 国家自然科学基金 (批准号: 10401038, 10171108, 10271002, 70271014) 和北京工业大学博士科研 启动基金及 NSERC 基金 (10004901) 资助项目

**E-mail: xudc@bjut.edu.cn 
DSP 可以化为下面的二次整数规划: 定义二值变量 $x$ : 如果 $i \in S, x_{i}=1$, 否 则取 -1 ,

$$
\begin{array}{ll}
\max & \frac{1}{4} \sum_{i<j} w_{i j}\left(1+x_{i}+x_{j}+x_{i} x_{j}\right) \\
\text { s.t. } & \sum_{j=1}^{n} x_{j}=0, \quad x_{j}^{2}=1, \quad j=1, \cdots, n .
\end{array}
$$

定义 1.2(表压缩问题 (TCP)) 设 $(A, B, E)$ 是一个二部图, 其中 $|A|=|B|=$ $n$ ( $n$ 是偶数). $A$ 和 $B$ 是两个分离的顶点集合, 边集 $E$ 中的边只连结 $A$ 和 $B$ 中的 点. 对边定义一个对称非负的权函数 $w: E \rightarrow \mathbb{R}^{+}$. 我们要寻找两个子集 $A_{1} \subset A$ 和 $B_{1} \subset B$, 使得 $\left(A_{1}, B_{1}\right)$ 之间的权和, 即 $\sum_{(i, j) \in E, i \in A_{1}, j \in B_{1}} w_{i j}$ 达到最大, 并且 $\left|A_{1}\right|=\left|B_{1}\right|=n / 2$.

类似地, 我们可以把 TCP 化为一个二次整数规划. 定义两个二值变量 $x$ 和 $y$ : 如果 $i \in A_{1}, x_{i}=1$, 否则取 -1 ; 如果 $j \in B_{1}, y_{j}=1$, 否则取 -1 .

$$
\begin{aligned}
w_{*}:=\max & \frac{1}{4} \sum_{i \in A, j \in B} w_{i j}\left(1+x_{i}+y_{j}+x_{i} y_{j}\right) \\
\text { s.t. } & \sum_{i \in A} x_{i}=0, \quad \sum_{j \in B} y_{j}=0, \\
& x_{i}^{2}=1, \quad i \in A, \quad y_{j}^{2}=1, \quad j \in B .
\end{aligned}
$$

本文考虑上述两个问题的近似算法. 目前对于 DSP 最好的近似比是 0.5886 (没 有三角不等式) 和 0.6236 (带三角不等式), 分别见文献 [1,2]. 后面的比值 0.6236 改 进了 Halperin 和 Zwick ${ }^{[3]}$ 给出的近似比 0.6221 . TCP 的最好比值是由简单贪婪算 法给出的 0.5. Anderson ${ }^{1)}$ 提出了如下未解决问题: 半定规划 (SDP) 是否可以改 进 0.5 的近似比. 我们在本文中从正面回答了该问题.

基于 SDP 松驰和改进的舍入技巧, 我们提出改进的近似算法. 本文的主要 结果是

(i) 对 DSP 提出一个 0.5982 - 近似算法;

(ii) 如果增加三角不等式, 我们得到 DSP 的 0.6243 - 近似算法;

(iii) 对 TCP 提出一个 0.5970 - 近似算法;

(iv) 如果增加三角不等式, 我们得到 TCP 的 0.6708 - 近似算法.

我们分别处理 DSP 和 TCP, 先给出一些 (弱的) 近似比, 然后通过增加三角不 等式加强 SDP 松弛来改进近似比, 最后做了一些讨论.

\section{DSP 的近似算法}

通过引入参考变量 $x_{0}$, 我们把 DSP 重新化为一个等价的齐次二次整数规划. 重新定义二值变量 $x$ : 如果 $i \in S, x_{i}=x_{0}$, 否则取 $-x_{0}$. 这样可以得到下面的 SDP

1) Anderson R. Combinatorial Optimization for Text Layout. University of Washington, 2000 
松弛:

$$
\begin{aligned}
w_{\mathrm{SDP}}:=\max & \frac{1}{4} \sum_{0<i<j} w_{i j}\left(1+X_{0 i}+X_{0 j}+X_{i j}\right) \\
\text { s.t. } & \sum_{j=1}^{n} X_{0 j}=0, \quad \sum_{i, j=1}^{n} X_{i j}=0, \\
& X_{j j}=1, \quad j=0, \cdots, n, \quad X \succeq 0 .
\end{aligned}
$$

\section{1 没有三角不等式约束的近似算法}

我们给出主算法, 本文的所有算法都是基于该算法. 除了步骤 2, 主算法与 文献 [1] 类似, 步骤 2 受文献 [4 7] 的启发.

给出 3 个参数 $\theta \in[0,1], \eta \in[-1,1], \gamma \in[0,+\infty)$, 我们在后面会把它们确定 下来.

\section{主算法}

1. 求解 SDP: 求解 (3) 式得到最优的半正定对称矩阵 $X^{*}$.

2. 随机舍入: 定义两个半正定矩阵

$$
I_{A}(\theta)=\left(\begin{array}{cc}
0 & 0 \\
0 & (1-\theta) I
\end{array}\right), \quad X^{*}(\theta)=\left(\begin{array}{cc}
X_{00}^{*} & \sqrt{\theta} X_{0 x}^{*} \\
\sqrt{\theta} X_{x 0}^{*} & \theta X_{x x}^{*}
\end{array}\right) .
$$

产生向量 $u$, 服从均值为 0 且协方差矩阵为 $X^{*}(\theta)+I_{A}(\theta)$ 的多元正态分布.

令 $\hat{x}=\operatorname{sign}(u)$, 即

$$
\hat{x}_{i}=\left\{\begin{array}{ll}
1, & \text { 如果 } u_{i} \geqslant 0, \\
-1, & \text { 如果 } u_{i}<0 .
\end{array} .\right.
$$

置 $S=\left\{i>0: \hat{x}_{i}=\hat{x}_{0}\right\}, \tilde{S}=S$.

3. 调整顶点:

- 如果 $|\tilde{S}|>n / 2$, 对每个 $i \in \tilde{S}$, 定义 $\zeta(i)=\sum_{j \in \tilde{S}} w_{i j}, \tilde{S}:=\left\{i_{1}, i_{2}, \cdots, i_{|\tilde{S}|}\right\}$, 其中 $\zeta\left(i_{1}\right) \geqslant \zeta\left(i_{2}\right) \geqslant \cdots \geqslant \zeta\left(i_{|\tilde{S}|}\right)$. 把顶点 $i_{|\tilde{S}|}$ 从集合 $\tilde{S}$ 中移出, 重新令 $\tilde{S}:=$ $\left\{i_{1}, i_{2}, \cdots, i_{|\tilde{S}|-1}\right\}$. 重复上述过程直到 $|\tilde{S}|=n / 2$.

- 如果 $|\tilde{S}| \leqslant n / 2$, 从 $\tilde{S}$ 外面任意增加 $\frac{n}{2}-|\tilde{S}|$ 个顶点到集合 $\tilde{S}$ 里.

我们引入一些记号. 设 $w(U)$ 为由集合 $U \subset V$ 导出的子图的所有权和. 我 们有

$$
w(\tilde{S}) \geqslant \begin{cases}\frac{\frac{n}{2}\left(\frac{n}{2}-1\right)}{|S|(|S|-1)} w(S), & \text { 如果 }|S|>n / 2, \\ w(S), & \text { 如果 }|S| \leqslant n / 2 .\end{cases}
$$

记

$$
\alpha(\theta)=\min _{(x, y, z) \in \mathcal{F}} \frac{1+\frac{2}{\pi}(\arcsin (\sqrt{\theta} x)+\arcsin (\sqrt{\theta} y)+\arcsin (\theta z))}{1+x+y+z},
$$




$$
\begin{gathered}
\beta(\theta)=1-\frac{2}{\pi} \arcsin \sqrt{\theta}+\min _{-1 \leqslant y<1} \frac{2}{\pi} \frac{\arcsin \sqrt{\theta}-\arcsin (\sqrt{\theta} y)}{1-y}, \\
\tilde{\beta}(\theta)=1-\frac{2}{\pi} \arcsin \theta+\min _{-1 \leqslant y<1} \frac{2}{\pi} \frac{\arcsin \theta-\arcsin (\theta y)}{1-y}, \\
\beta_{1}= \begin{cases}\beta(\theta) / 2, & \text { 如果 } \eta \geqslant 0, \\
\frac{2-\beta(\theta)}{2}, & \text { 如果 } \eta<0, \\
\beta_{2}=\frac{n \tilde{\beta}(\theta)-\left(1-\frac{2}{\pi} \arcsin \theta\right)}{4 n},\end{cases}
\end{gathered}
$$

其中

$$
\mathcal{F}:=\left\{(x, y, z) \mid 1+x+y+z>0,\left(\begin{array}{ccc}
1 & x & y \\
x & 1 & z \\
y & z & 1
\end{array}\right) \succeq 0\right\} .
$$

定义下面的随机变量:

$$
\begin{gathered}
w:=w(S)=\frac{1}{4} \sum_{0<i<j} w_{i j}\left(1+\hat{x}_{0} \hat{x}_{i}+\hat{x}_{0} \hat{x}_{j}+\hat{x}_{i} \hat{x}_{j}\right), \\
p:=|S|=\frac{1}{2} \sum_{j>0}\left(1+\hat{x}_{0} \hat{x}_{j}\right), \\
m:=|S|(n-|S|)=\frac{1}{4} \sum_{i>0, j>0}\left(1-\hat{x}_{i} \hat{x}_{j}\right), \\
z(\eta, \gamma):=\frac{w}{w_{*}}+\eta \gamma \frac{p}{n}+\gamma \frac{m}{n^{2}} .
\end{gathered}
$$

下面分析算法. 主算法给出 $S$ 满足不等式:

$$
E[w] \geqslant \alpha w_{*}, \quad E[\eta p / n] \geqslant \eta \beta_{1}, \quad E\left[m / n^{2}\right] \geqslant \beta_{2},
$$

从而有 $E[z(\eta, \gamma)] \geqslant \mu_{\eta, \gamma}:=\alpha+\gamma\left(\eta \beta_{1}+\beta_{2}\right)$. 限制 $\eta \in[-1,0]$, 令

$$
\gamma= \begin{cases}\frac{4 \alpha}{1-4\left(\eta \beta_{1}+\beta_{2}\right)}, & \text { 如果 } \eta \in[-1 / 2,0], \\ \frac{3 \alpha}{\left(\eta \beta_{1}+\beta_{2}\right)-\eta+(1+\eta)^{2}-4\left(\eta \beta_{1}+\beta_{2}\right)}, & \text { 如果 } \eta \in[-1,-1 / 2) .\end{cases}
$$

Han 等 ${ }^{[8]}$ 证明了

引理 2.1 如果随机变量 $z(\eta, \gamma)$ 超过它的均值, 即 $z(\eta, \gamma) \geqslant \mu_{\eta, \gamma}$, 则 $w(\tilde{S}) \geqslant$ $R(\eta, \theta) w^{*}$, 其中

$$
R(\eta, \theta)= \begin{cases}\frac{\alpha\left(1-(1+\eta)^{2}\right)}{1-4\left(\eta \beta_{1}+\beta_{2}\right)}, & \text { 如果 } \eta \in[-1 / 2,0], \\ \frac{\alpha\left((1+\eta)^{2}-4 \eta\right) / 4}{\left(\eta \beta_{1}+\beta_{2}\right)-\eta+(1+\eta)^{2}-4\left(\eta \beta_{1}+\beta_{2}\right)}, & \text { 如果 } \eta \in[-1,-1 / 2) .\end{cases}
$$


最后求解极大化问题

$$
\begin{aligned}
R=\max & R(\eta, \theta) \\
\text { s.t. } & 0 \leqslant \theta \leqslant 1, \quad-1 \leqslant \eta \leqslant 0 .
\end{aligned}
$$

利用 Matlab 6.0, 计算得到 $R=0.5982$, 这时 $\theta=0.82, \eta=-0.1434, \alpha(\theta)=$ $0.7276, \beta(\theta)=0.9581, \gamma=8.9869$. 结合引理 2.1 和 Frieze 与 Jerrum ${ }^{[9]}$ 的分析, 我 们有

定理 2.2 对于 DSP, 主算法的最坏情形下的近似比是 0.5982 .

\section{2 有三角不等式约束的改进近似算法}

现在将映射 (map) 技巧 ${ }^{[10]}$ 和线性随机舍入 (LRR) ${ }^{[3]}$ 结合到主算法中, 从而 得到更好的近似比. 新导出的算法记为主-Dsp-Map-Lrr 算法. 我们通过增加所谓 的三角不等式 (4) (7) 来加强 SDP 松他 (3). 记导出的新 SDP 松弛为 Dsp-TriangleSdp.

$$
\begin{aligned}
X_{i j}+X_{i k}+X_{j k} \geqslant-1, & 0 \leqslant i, j, k \leqslant n, \\
-X_{i j}-X_{i k}+X_{j k} \geqslant-1, & 0 \leqslant i, j, k \leqslant n, \\
-X_{i j}+X_{i k}-X_{j k} \geqslant-1, & 0 \leqslant i, j, k \leqslant n, \\
X_{i j}-X_{i k}-X_{j k} \geqslant-1, & 0 \leqslant i, j, k \leqslant n .
\end{aligned}
$$

对主算法进行下面的调整得到改进的主 -Dsp-Map-Lrr 算法:

- 在步 1 中, 求解 Dsp-Triangle-Sdp 而不是 (3) 式.

- 在步 1 后面, 增加映射步:

映射: 设

$$
X^{*}=\left[v_{0}, v_{1}, \cdots, v_{n}\right]^{\mathrm{T}}\left[v_{0}, v_{1}, \cdots, v_{n}\right]
$$

其中 $v_{i} \in \mathbb{R}^{n+1},\left\|v_{i}\right\|=1, i=0,1, \cdots, n$. 将向量 $v_{i}$ 映射到依赖于 $v_{0}$ 和 $v_{i}$ 的向量 $w_{i}$. 记 $w_{0}:=v_{0}$. 置

$$
X^{*}=\left[w_{0}, w_{1}, \cdots, w_{n}\right]^{\mathrm{T}}\left[w_{0}, w_{1}, \cdots, w_{n}\right] .
$$

- 在步 2 后面, 增加下面的步骤:

线性舍入: 以概率 $\nu$ 重新选取 $S$, 分别以概率 $\left(1+v_{0} \cdot v_{i}\right) / 2$ 和 $\left(1-v_{0} \cdot v_{i}\right) / 2$ 把每个 $i \in V$ 独立地选入 $S$ 和 $V \backslash S$.

在分析主 -Dsp-Map-Lrr 算法之前, 我们简单地解释一下映射和线性随机舍 入的动机. 对这两个技巧的详细讨论, 可以参见文献 [10,3]. 映射的思想是旋转 向量, 使得它与向量 $v_{0}$ 或 $-v_{0}$ 更靠近, 从而可以比较容易地从 SDP 的解舍入为 一个整数解. 直观上, 我们认为与 $v_{0}$ 靠近的向量应选入 $S$. 线性随机舍入是增加 随机步骤来舍入这些向量.

下面分析改进的算法. 把一些记号的定义修改一下, 分析与 2.1 节相同. 定义映射函数 $f(x ; \rho)$ (参见文献 $[10]), f(x ; \rho):=(1-\rho) x+\rho\left[\frac{\pi}{2}(1-\cos x)\right]$, 这里 
$\rho \in[0,1]$ 是参数. 重新定义 $\alpha(\theta), \beta(\theta), \tilde{\beta}(\theta), \beta_{1}, \beta_{2}$, 其他的记号与 2.1 节一样:

$$
\begin{gathered}
\alpha(\theta)=\min _{(x, y, z) \in \mathcal{F}} \frac{(1-\nu) \tilde{f}(x, y, z)+\nu(1+x)(1+y)}{1+x+y+z}, \\
\beta(\theta)=1-\frac{2}{\pi} \arcsin \sqrt{\theta}+\min _{-1 \leqslant y<1} \frac{2}{\pi} \frac{\arcsin \sqrt{\theta}-\arcsin (\sqrt{\theta} \cos f(\arccos y))}{1-y}, \\
\tilde{\beta}(\theta)=1-\frac{2}{\pi} \arcsin \theta \\
+\min _{(x, y, z) \in \mathcal{F}} \frac{2}{\pi} \frac{\arcsin \theta-\arcsin (\theta g(\arccos x, \arccos y, \arccos z))}{1-z}, \\
\beta_{1}= \begin{cases}\frac{(1-\nu) \beta(\theta)+\nu}{2}, & \text { 如果 } \eta \geqslant 0, \\
\frac{(1-\nu)(2-\beta(\theta))+\nu}{2}, & \text { 如果 } \eta<0, \\
\beta_{2}=\frac{(1-\nu)\left[n \tilde{\beta}(\theta)-\left(1-\frac{2}{\pi} \arcsin \theta\right)\right]+\nu(n-2)}{4 n},\end{cases}
\end{gathered}
$$

其中

$$
\begin{aligned}
& \tilde{f}(x, y, z)=1+\frac{2}{\pi}[\arcsin (\sqrt{\theta} \cos f(\arccos x))+\arcsin (\sqrt{\theta} \cos f(\arccos y)) \\
& +\arcsin (\theta g(\arccos x, \arccos y, \arccos z))], \\
& g(x, y, z)=\cos f(x) \cos f(y)+\frac{\cos z-\cos x \cos y}{\sin x \sin y} \sin f(x) \sin f(y),
\end{aligned}
$$

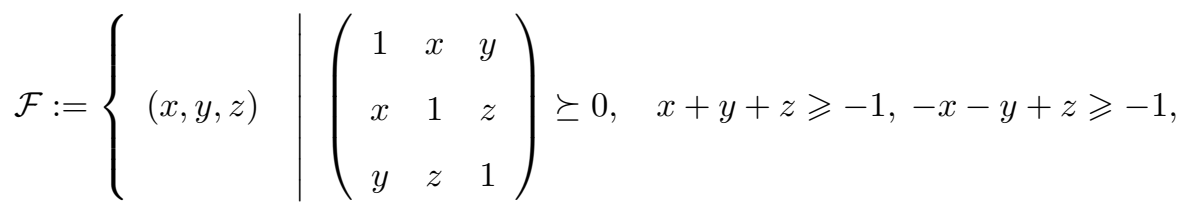

$$
\begin{aligned}
& -x+y-z \geqslant-1, x-y-z \geqslant-1\} .
\end{aligned}
$$

当 $\theta=0.87, \rho=0.22, \nu=0.13, \eta=-0.1769, \gamma=7.7442$ 时, 我们得到 $\alpha(\theta)=0.7765, \beta(\theta)=0.9727, \tilde{\beta}(\theta)=0.9553, R=0.6243$.

定理 2.3 对于 DSP, 主 -Dsp-Map-Lrr 算法在最坏情形下的近似比是 0.6243 (有三角不等式约束).

\section{TCP 的近似算法}

本节处理 TCP. 与前面类似, 我们首先应用标准的 SDP 松驰获得较弱的比 值, 然后通过增加三角不等式来改进这些比值. TCP(2) 的 SDP 松弛为

$$
w_{\mathrm{SDP}}:=\max \frac{1}{4} \sum_{1 \leqslant i \leqslant n, n+1 \leqslant j \leqslant 2 n} w_{i j}\left(1+U_{0 i}+U_{0 j}+U_{i j}\right)
$$




$$
\begin{array}{ll}
\text { s.t. } & \sum_{i=1}^{n} U_{0 i}=0, \quad \sum_{i=n+1}^{2 n} U_{0 i}=0, \\
& \sum_{1 \leqslant i, j \leqslant n} U_{i j}=0, \quad \sum_{n+1 \leqslant i, j \leqslant 2 n} U_{i j}=0, \\
& U_{i i}=1, \quad i=0,1, \cdots, 2 n, \quad U \succeq 0 .
\end{array}
$$

除了顶点调整步骤 (步 3) 外, 我们对 TCP 给出的算法与主算法类似. 下面 指出它们的区别. 在本节中, 除非特别声明, 所有的记号与 2.1 小节相同.

\section{1 没有三角不等式约束的近似算法}

对 TCP 给出的算法记为主 -Tcp 算法, 它与主算法类似, 只需要进行下面的 修改:

- 步 1 , 我们求解 (8) 式而不是 (3) 式.

- 步 3 由下面的贪婪算法代替:

贪婪调整: 设在主算法的步 2 之后, 选取 $S_{1} \subset A$ 和 $S_{2} \subset B$. 记

$$
\tilde{S}_{1}=S_{1}, \quad \tilde{S}_{2}=S_{2} .
$$

情形 $1 \quad\left|\tilde{S}_{1}\right|>n / 2,\left|\tilde{S}_{2}\right|>n / 2$. 对每个 $i \in \tilde{S}_{1}$, 定义 $\zeta(i)=\sum_{j \in \tilde{S}_{2}} w_{i j}$, 置 $\tilde{S}_{1}:=\left\{i_{1}, i_{2}, \cdots, i_{\left|\tilde{S}_{1}\right|}\right\}$, 其中 $\zeta\left(i_{1}\right) \geqslant \zeta\left(i_{2}\right) \geqslant \cdots \geqslant \zeta\left(i_{\left|\tilde{S}_{1}\right|}\right)$. 把顶点 $i_{\left|\tilde{S}_{1}\right|}$ 从 $\tilde{S}_{1}$ 中移 出, 置 $\tilde{S}_{1}:=\left\{i_{1}, i_{2}, \cdots, i_{\left|\tilde{S}_{1}\right|-1}\right\}$. 重复此调整过程直到 $\left|\tilde{S}_{1}\right|=n / 2$. 用同样的办法 处理 $\tilde{S}_{2}$, 使得 $\left|\tilde{S}_{2}\right|=n / 2$.

情形 $2\left|\tilde{S}_{1}\right|>n / 2,\left|\tilde{S}_{2}\right| \leqslant n / 2$. 与情形 1 类似处理 $\tilde{S}_{1}$, 使得 $\left|\tilde{S}_{1}\right|=n / 2$. 从 $B \backslash \tilde{S}_{2}$ 中任意取 $\frac{n}{2}-\left|\tilde{S}_{2}\right|$ 个顶点加入到 $\tilde{S}_{2}$ 中.

情形 $3\left|\tilde{S}_{1}\right| \leqslant n / 2,\left|\tilde{S}_{2}\right|>n / 2$. 与情形 2 类似处理.

情形 $4\left|\tilde{S}_{1}\right| \leqslant n / 2,\left|\tilde{S}_{2}\right| \leqslant n / 2$. 从 $A \backslash \tilde{S}_{1}$ 中任意取 $\frac{n}{2}-\left|\tilde{S}_{1}\right|$ 个顶点加入到 $\tilde{S}_{1}$ 中, 从 $B \backslash \tilde{S}_{2}$ 中任意取 $\frac{n}{2}-\left|\tilde{S}_{2}\right|$ 个顶点加入到 $\tilde{S}_{2}$ 中.

本小节下面的内容如下: 先给出主 -Tcp 算法的初步分析 (3.1.1), 然后通过加 入更多的信息得到更好的结果 (3.1.2 和 3.1.3), 从而一步步地给出改进的近似比.

3.1.1 初步的分析 我们对前面的贪婪算法进行分析. 首先有

$$
w\left(\tilde{S}_{1}, \tilde{S}_{2}\right) \geqslant \begin{cases}\frac{n}{2\left|S_{1}\right|} \cdot \frac{n}{2\left|S_{2}\right|} \cdot w\left(S_{1}, S_{2}\right), & \text { 如果 }\left|S_{1}\right|>n / 2,\left|S_{2}\right|>n / 2 ; \\ \frac{n}{2\left|S_{1}\right|} \cdot w\left(S_{1}, S_{2}\right), & \text { 如果 }\left|S_{1}\right|>n / 2,\left|S_{2}\right| \leqslant n / 2 ; \\ \frac{n}{2\left|S_{2}\right|} \cdot w\left(S_{1}, S_{2}\right), & \text { 如果 }\left|S_{1}\right| \leqslant n / 2,\left|S_{2}\right|>n / 2 ; \\ w\left(S_{1}, S_{2}\right), & \text { 如果 }\left|S_{1}\right| \leqslant n / 2,\left|S_{2}\right| \leqslant n / 2 .\end{cases}
$$

在上述 4 种情形中, 我们只考虑第 1 种情形, 原因是它对 $w\left(\tilde{S}_{1}, \tilde{S}_{2}\right)$ 给出最小下 界 $\frac{n}{2\left|S_{1}\right|} \cdot \frac{n}{2\left|S_{2}\right|} \cdot w\left(S_{1}, S_{2}\right)$.

定义

$$
w:=w\left(S_{1}, S_{2}\right), \quad m_{1}:=\left|S_{1}\right|\left(n-\left|S_{1}\right|\right), \quad m_{2}:=\left|S_{2}\right|\left(n-\left|S_{2}\right|\right),
$$




$$
z=\frac{w}{w_{*}}+\gamma \frac{m_{1}+m_{2}}{n^{2}}
$$

我们有 (参见文献 [1])

$$
E[w] \geqslant \alpha(\theta) \cdot w_{*}, \quad E\left[m_{i} / n^{2}\right] \geqslant \beta_{2} \quad(i=1,2), \quad E[z] \geqslant \mu_{\gamma}:=\alpha+2 \gamma \beta_{2} .
$$

记

$$
w\left(S_{1}, S_{2}\right)=\lambda w_{*}, \quad\left|S_{1}\right|=\delta_{1} n, \quad\left|S_{2}\right|=\delta_{2} n,
$$

则

$$
\begin{gathered}
\lambda \geqslant \mu-\gamma \delta_{1}\left(1-\delta_{1}\right)-\gamma \delta_{2}\left(1-\delta_{2}\right) \\
w\left(\tilde{S}_{1}, \tilde{S}_{2}\right) \geqslant \frac{w\left(S_{1}, S_{2}\right)}{4 \delta_{1} \delta_{2}}=\frac{\lambda w_{*}}{4 \delta_{1} \delta_{2}} \geqslant \frac{\mu-\gamma \delta_{1}\left(1-\delta_{1}\right)-\gamma \delta_{2}\left(1-\delta_{2}\right)}{4 \delta_{1} \delta_{2}} \cdot w_{*} .
\end{gathered}
$$

定义

$$
\hat{f}\left(\delta_{1}, \delta_{2}\right)=\frac{\mu-\gamma \delta_{1}\left(1-\delta_{1}\right)-\gamma \delta_{2}\left(1-\delta_{2}\right)}{\delta_{1} \delta_{2}},
$$

则

$$
\hat{f}_{\delta_{1}}^{\prime}=\frac{-\mu+\gamma \delta_{2}\left(1-\delta_{2}\right)+\gamma \delta_{1}^{2}}{\delta_{2} \delta_{1}^{2}}, \quad \hat{f}_{\delta_{2}}^{\prime}=\frac{-\mu+\gamma \delta_{1}\left(1-\delta_{1}\right)+\gamma \delta_{2}^{2}}{\delta_{1} \delta_{2}^{2}}
$$

考虑系统

$$
\left\{\begin{array}{l}
\hat{f}_{\delta_{1}}^{\prime}=0 \\
\hat{f}_{\delta_{2}}^{\prime}=0
\end{array}\right.
$$

或

$$
\left\{\begin{array}{c}
\delta_{2}\left(1-\delta_{2}\right)+\delta_{1}^{2}=\mu / \gamma \\
\delta_{1}\left(1-\delta_{1}\right)+\delta_{2}^{2}=\mu / \gamma
\end{array}\right.
$$

它的解为 $\delta_{1}=\delta_{2}=\delta_{0}:=\mu / \gamma$. 定义 $\hat{f}_{0}:=\hat{f}\left(\delta_{0}, \delta_{0}\right)$, 考虑

$$
\begin{aligned}
\hat{f}\left(\delta_{1}, 1\right) & =\frac{\mu-\gamma \delta_{1}\left(1-\delta_{1}\right)}{\delta_{1}}=\frac{\mu}{\delta_{1}}+\gamma \delta_{1}-\gamma \geqslant R_{1}(\gamma ; \theta):=2 \sqrt{\gamma \mu}-\gamma, \\
\hat{f}\left(\delta_{1}, \frac{1}{2}\right) & =\frac{2 \mu-2 \gamma \delta_{1}\left(1-\delta_{1}\right)-\frac{\gamma}{2}}{\delta_{1}}=\frac{4 \mu-\gamma}{2 \delta_{1}}+2 \gamma \delta_{1}-2 \gamma \geqslant R_{2}(\gamma ; \theta) \\
& :=2 \sqrt{\gamma(4 \mu-\gamma)}-2 \gamma .
\end{aligned}
$$

令

$$
R(\theta):= \begin{cases}\max _{\gamma>0} \frac{1}{4} \min \left\{f_{0}, R_{1}(\gamma ; \theta), R_{2}(\gamma ; \theta)\right\}, & \text { 如果 } \delta_{0} \in[1 / 2,1], \\ \max _{\gamma>0} \frac{1}{4} \min \left\{R_{1}(\gamma ; \theta), R_{2}(\gamma ; \theta)\right\}, & \text { 如果 } \delta_{0} \in[0,1 / 2] .\end{cases}
$$

这表明 $4 R(\theta)$ 是 $\hat{f}\left(\delta_{1}, \delta_{2}\right)$ 当 $\left(\delta_{1}, \delta_{2}\right) \in[1 / 2,1 ; 1 / 2,1]$ 的最小值. 
当 $\theta=0.75, \gamma=9.4729$ 时, 我们有 $\alpha(\theta)=0.6967, \tilde{\beta}(\theta)=0.9831, R=0.5455$, 因此主 -Tcp 算法在最坏情形下的近似比为 0.5455 .

3.1.2 加人 $\left(n-\left|S_{1}\right|\right)\left(n-\left|S_{2}\right|\right)$ 项 通过加入 $\left(n-\left|S_{1}\right|\right)\left(n-\left|S_{2}\right|\right)$ 项, 我们 来改进前面的比值. 定义

$$
\begin{aligned}
m_{3} & :=\left(n-\left|S_{1}\right|\right)\left(n-\left|S_{2}\right|\right)=\frac{1}{2} \sum_{i>0}\left(1-\hat{x}_{0} \hat{x}_{i}\right) \frac{1}{2} \sum_{j>0}\left(1-\hat{x}_{0} \hat{y}_{j}\right) \\
& =\frac{1}{4} \sum_{i>0, j>0}\left(1-\hat{x}_{0} \hat{x}_{i}-\hat{x}_{0} \hat{y}_{j}+\hat{x}_{i} \hat{y}_{j}\right) .
\end{aligned}
$$

记

$$
\alpha^{\prime}(\theta)=\min _{(x, y, z) \in \mathcal{F}^{\prime}} \frac{1+\frac{2}{\pi}(-\arcsin (\sqrt{\theta} x)-\arcsin (\sqrt{\theta} y)+\arcsin (\theta z))}{1-x-y+z},
$$

其中

$$
\mathcal{F}^{\prime}:=\left\{(x, y, z) \mid 1-x-y+z>0,\left(\begin{array}{ccc}
1 & x & y \\
x & 1 & z \\
y & z & 1
\end{array}\right) \succeq 0\right\} .
$$

容易看出 $\alpha^{\prime}(\theta)=\alpha(\theta)$, 从而 $E\left[m_{3} / n^{2}\right] \geqslant \alpha^{\prime} / 4=\alpha / 4$. 定义

$$
z=\frac{w}{w_{*}}+\gamma \frac{m_{1}+m_{2}+m_{3}}{n^{2}},
$$

则

$$
\begin{gathered}
E[z] \geqslant \mu_{\gamma}:=\alpha+\gamma\left(2 \beta_{2}+\frac{\alpha}{4}\right), \\
\lambda \geqslant \mu-\gamma \delta_{1}\left(1-\delta_{1}\right)-\gamma \delta_{2}\left(1-\delta_{2}\right)-\gamma\left(1-\delta_{1}\right)\left(1-\delta_{2}\right) .
\end{gathered}
$$

记

$$
\hat{f}\left(\delta_{1}, \delta_{2}\right)=\frac{\mu-\gamma \delta_{1}\left(1-\delta_{1}\right)-\gamma \delta_{2}\left(1-\delta_{2}\right)-\gamma\left(1-\delta_{1}\right)\left(1-\delta_{2}\right)}{\delta_{1} \delta_{2}} .
$$

我们有

$$
\hat{f}_{\delta_{1}}^{\prime}=\frac{-\mu+\gamma\left(1-\delta_{2}^{2}+\delta_{1}^{2}\right)}{\delta_{1}^{2} \delta_{2}}, \quad \hat{f}_{\delta_{2}}^{\prime}=\frac{-\mu+\gamma\left(1-\delta_{1}^{2}+\delta_{2}^{2}\right)}{\delta_{1} \delta_{2}^{2}} .
$$

方程组

$$
\left\{\begin{array} { l } 
{ \hat { f } _ { \delta _ { 1 } } ^ { \prime } = 0 , } \\
{ \hat { f } _ { \delta _ { 2 } } ^ { \prime } = 0 }
\end{array} \text { 或 } \quad \left\{\begin{array}{l}
1-\delta_{2}^{2}+\delta_{1}^{2}=\mu / \gamma, \\
1-\delta_{1}^{2}+\delta_{2}^{2}=\mu / \gamma
\end{array}\right.\right.
$$

对 $\left(\delta_{1}, \delta_{2}\right) \in[0,1 ; 0,1]$ 无解 (我们加入约束 $\gamma \neq \mu$ ). 考虑

$$
\begin{aligned}
\hat{f}\left(\delta_{1}, 1\right) & =\frac{\mu-\gamma \delta_{1}\left(1-\delta_{1}\right)}{\delta_{1}}=\frac{\mu}{\delta_{1}}+\gamma \delta_{1}-\gamma \\
& \geqslant R_{1}(\gamma ; \theta):=2 \sqrt{\gamma \mu}-\gamma,
\end{aligned}
$$

www.scichina.com 


$$
\begin{aligned}
\hat{f}\left(\delta_{1}, 1 / 2\right) & =\frac{2 \mu-2 \gamma \delta_{1}\left(1-\delta_{1}\right)-\frac{\gamma}{2}-\gamma\left(1-\delta_{1}\right)}{\delta_{1}} \\
& =\frac{4 \mu-3 \gamma}{2 \delta_{1}}+2 \gamma \delta_{1}-\gamma \\
& \geqslant R_{2}(\gamma ; \theta):=2 \sqrt{\gamma(4 \mu-3 \gamma)}-\gamma .
\end{aligned}
$$

记 $R(\theta)=\max _{\gamma \geqslant 0} \frac{1}{4} \min \left\{R_{1}(\gamma ; \theta), R_{2}(\gamma ; \theta)\right\}$. 当 $\theta=0.95, \gamma=2.3286$ 时, 有 $\alpha(\theta)=$ $0.7785, \tilde{\beta}(\theta)=0.9421, R=0.5822$.

3.1.3 增加参数 $\eta$ 在分析中引入新的参数 $\eta$, 我们可以改进前面的比值. 定 义 $p_{1}:=\left|S_{1}\right|$ 和 $p_{2}:=\left|S_{2}\right|$, 则 $E\left[\eta p_{i} / n\right] \geqslant \eta \beta_{1}, i=1,2$.

对新的随机变量

$$
z(\eta, \gamma):=\frac{w}{w_{*}}+\eta \gamma \frac{p_{1}+p_{2}}{n}+\gamma \frac{m_{1}+m_{2}+m_{3}}{n^{2}},
$$

我们有

$$
\begin{gathered}
E[z] \geqslant \mu_{\eta, \gamma}:=\alpha+\gamma\left(2 \eta \beta_{1}+2 \beta_{2}+\frac{\alpha}{4}\right), \\
\lambda \geqslant \mu-\eta \gamma\left(\delta_{1}+\delta_{2}\right)-\gamma \delta_{1}\left(1-\delta_{1}\right)-\gamma \delta_{2}\left(1-\delta_{2}\right)-\gamma\left(1-\delta_{1}\right)\left(1-\delta_{2}\right) .
\end{gathered}
$$

记

$$
\begin{gathered}
\hat{f}\left(\delta_{1}, \delta_{2}\right)=\frac{\mu-\eta \gamma\left(\delta_{1}+\delta_{2}\right)-\gamma \delta_{1}\left(1-\delta_{1}\right)-\gamma \delta_{2}\left(1-\delta_{2}\right)-\gamma\left(1-\delta_{1}\right)\left(1-\delta_{2}\right)}{\delta_{1} \delta_{2}}, \\
\hat{f}_{\delta_{1}}^{\prime}=\frac{-\mu+\eta \gamma \delta_{2}+\gamma\left(1-\delta_{2}^{2}+\delta_{1}^{2}\right)}{\delta_{1}^{2} \delta_{2}}, \quad \hat{f}_{\delta_{2}}^{\prime}=\frac{-\mu+\eta \gamma \delta_{1}+\gamma\left(1-\delta_{1}^{2}+\delta_{2}^{2}\right)}{\delta_{1} \delta_{2}^{2}},
\end{gathered}
$$

求解方程组

$$
\left\{\begin{array} { l } 
{ \hat { f } _ { \delta _ { 1 } } ^ { \prime } = 0 , } \\
{ \hat { f } _ { \delta _ { 2 } } ^ { \prime } = 0 }
\end{array} \text { 或 } \quad \left\{\begin{array}{l}
\eta \delta_{2}+1-\delta_{2}^{2}+\delta_{1}^{2}=\mu / \gamma, \\
\eta \delta_{1}+1-\delta_{1}^{2}+\delta_{2}^{2}=\mu / \gamma,
\end{array}\right.\right.
$$

得到 $\delta_{1}=\delta_{2}=\delta_{0}:=\frac{\mu-\gamma}{\eta \gamma}$. 记 $\hat{f}_{0}:=\hat{f}\left(\delta_{0}, \delta_{0}\right)$. 考虑

$$
\begin{gathered}
\hat{f}\left(\delta_{1}, 1\right)=\frac{\mu-\eta \gamma}{\delta_{1}}+\gamma \delta_{1}-\eta \gamma-\gamma \\
\geqslant R_{1}(\eta, \gamma ; \theta):=2 \sqrt{\gamma(\mu-\eta \gamma)}-\eta \gamma-\gamma, \\
\hat{f}\left(\delta_{1}, 1 / 2\right)=\frac{4 \mu-2 \eta \gamma-3 \gamma}{2 \delta_{1}}+2 \gamma \delta_{1}-2 \eta \gamma-\gamma \\
\geqslant R_{2}(\eta, \gamma ; \theta):=2 \sqrt{\gamma(4 \mu-2 \eta \gamma-3 \gamma)}-2 \eta \gamma-\gamma .
\end{gathered}
$$

记

$$
R(\theta):= \begin{cases}\max _{-1 \leqslant \eta \leqslant 1, \gamma>0} \frac{1}{4} \min \left\{\hat{f}_{0}, R_{1}(\eta, \gamma ; \theta), R_{2}(\eta, \gamma ; \theta)\right\}, & \text { 如果 } \delta_{0} \in[1 / 2,1], \\ \max _{-1 \leqslant \eta \leqslant 1, \gamma>0} \frac{1}{4} \min \left\{R_{1}(\eta, \gamma ; \theta), R_{2}(\eta, \gamma ; \theta)\right\}, & \text { 如果 } \delta_{0} \in[0,1 / 2] .\end{cases}
$$

当 $\theta=0.93, \eta=-0.5462, \gamma=1.3816$ 时, 我们有 $\alpha(\theta)=0.7712, R=0.5970$. 
定理 3.1 主-Tcp 算法在最坏情形下的近似比为 0.5970 .

\section{2 有三角不等式约束的改进近似算法}

与 DSP 一样, 我们增加三角不等式来加强 TCP 的 SDP 松他 (8) 式. 新的 SDP 松弛记为 Tcp-Triangle-Sdp. 本小节的算法和 3.1 小节的主 -Tcp 算法类似, 除了下 面的修改:

- 求解 Tcp-Triangle-Sdp 而不是 (8) 式.

- 在步 1 后面, 增加映射步:

设 $U^{*}$ 是 Tcp-Triangle-Sdp 的最优解. 置

$$
U^{*}=\left[v_{0}, v_{1}, \cdots, v_{n}, u_{1}, \cdots, u_{n}\right]^{\mathrm{T}}\left[v_{0}, v_{1}, \cdots, v_{n}, u_{1}, \cdots, u_{n}\right],
$$

其中

$$
v_{i}, u_{i} \in \mathbb{R}^{2 n+1}, \quad\left\|v_{0}\right\|=\left\|v_{i}\right\|=\left\|u_{i}\right\|=1, \quad i=1, \cdots, n .
$$

映射 $v_{i}$ (或 $\left.u_{i}\right)$ 到依赖于 $v_{0}$ 和 $v_{i}$ 的向量 $v_{i}^{\prime}$ (或 $u_{i}^{\prime}$. 置

$$
U^{*}=\left[v_{0}, v_{1}^{\prime}, \cdots, v_{n}^{\prime}, u_{1}^{\prime}, \cdots, u_{n}^{\prime}\right]^{\mathrm{T}}\left[v_{0}, v_{1}^{\prime}, \cdots, v_{n}^{\prime}, u_{1}^{\prime}, \cdots, u_{n}^{\prime}\right] .
$$

- 增加线性舍入步骤如下:

顶点 $i \in A$ 以概率 $\left(1+v_{0} \cdot v_{i}\right) / 2$ 选入 $A_{1}$, 以概率 $\left(1-v_{0} \cdot v_{i}\right) / 2$ 选入 $A \backslash A_{1}$; 顶点 $i \in B$ 以概率 $\left(1+v_{0} \cdot u_{i}\right) / 2$ 选入 $B_{1}$, 以概率 $\left(1-v_{0} \cdot u_{i}\right) / 2$ 选入 $B \backslash B_{1}$. 不同 顶点的选取是独立的.

上述算法记为主 -Tsp-Map-Lrr 算法. 除了一些记号需要修改外, 分析和前面 的相同. 给定 $\theta, \rho, \nu \in[0,1], \alpha, \beta, \tilde{\beta}, \beta_{1}, \beta_{2}$ 与 2.2 小节相同. 当 $\theta=0.95, \rho=$ $0.45, \nu=0.1, \eta=-0.9812, \gamma=1.1625$ 时, 我们有 $\alpha(\theta)=0.8219, R=0.6708$.

定理 3.2 主 -Tcp-Map-Lrr 算法在最坏情形下的近似比为 0.6708 (带三角不 等式约束).

\section{4 讨论}

本文考虑两个 NP 问题: DSP 和 TCP. 对每一个问题，我们提出了基于 SDP 松能的改进的近似算法和改进的舍入技巧. 同时, 顺便回答了 Anderson 提出的 一个问题. 实际上, 我们对 DSP 提出的算法 (主算法的步骤 2) 对于改进其他的图 划分问题 (参见文献 [8]) 的近似比也是有帮助的. 我们提出如下问题作为下一步 的研究方向：改进本文的近似比，最好同时给出问题的近似比的上界.

致谢 感谢叶荫宇教授和张家伟教授的讨论和建议.

\section{参 考文献}

1 Ye Y, Zhang J. Approximation of Dense- $\frac{n}{2}$-Subgraph and the complement of Min-Bisection. Journal of Global Optimization, 2003, 25: 55 73 
$2 \mathrm{Xu} \mathrm{D}$, Han J, Huang Z, et al. Improved approximation algorithms for MAX $\frac{n}{2}$-DIRECTED-BISECTION and MAX $\frac{n}{2}$-DENSE-SUBGRAPH. Journal of Global Optimization, 2003, 27: 399 410

3 Halperin E, Zwick U. A unified framework for obtaining improved approximation algorithms for maximum graph bisection problems. Random Structures and Algorithms, 2002, 20(3): 382 402

4 Goemans M X, Williamson D P. Improved approximation algorithms for maximum cut and satisfiability problems using semidefinite programming. Journal of ACM, 1995, 42: 1115 1145

5 Zwick U. Outward rotations: a tool for rounding solutions of semidefinite programming relaxations, with applications to max cut and other problems. In: Proceedings of the 30th Symposium on Theory of Computation (STOC), 1999. 679 687

6 Ye Y. A .699-approximation algorithm for Max-Bisection. Math Programming, 2001, 90: 101 111

$7 \mathrm{Xu}$ D, Ye Y, Zhang J. Approximate the 2-catalog segmentation problem using semidefinite programming relaxation. Optimization Method and Software, 2003, 18: 705 719

8 Han Q, Ye Y, Zhang J. An improved rounding method and semidefinite programming relaxation for graph partition. Math Programming, 2002, 92: 509 535

9 Frieze A, Jerrum M. Improved approximation algorithms for max $k$-cut and max bisection. Algorithmica, 1997, 18: $67 \sim 81$

10 Feige U, Goemans M X. Approximating the value of two prover proof systems, with applications to MAX 2SAT and MAX DICUT. In: Proceedings of the 3rd Israel Symposium on Theory and Computing Systems, Tel Aviv, Israel, 1995. 182 189 